\title{
Impact of hydrogel peri-rectal spacer insertion on prostate gland intra-fraction motion during 1.5 T MR-guided stereotactic body radiotherapy
}

Francesco Cuccia ${ }^{1 *}$ (D), Rosario Mazzola ${ }^{1}$, Luca Nicosia ${ }^{1}$, Vanessa Figlia ${ }^{1}$, Niccolò Giaj-Levra ${ }^{1}$, Francesco Ricchetti ${ }^{1}$, Michele Rigo ${ }^{1}$, Claudio Vitale ${ }^{1}$, Beatrice Mantoan ${ }^{1}$, Antonio De Simone ${ }^{1}$, Gianluisa Sicignano ${ }^{1}$, Ruggero Ruggieri ${ }^{1}$, Stefano Cavalleri ${ }^{2}$ and Filippo Alongi, ${ }^{1,3}$

\begin{abstract}
Background: The assessment of organ motion is a crucial feature for prostate stereotactic body radiotherapy (SBRT). Rectal spacer may represent a helpful device in order to outdistance rectal wall from clinical target, but its impact on organ motion is still a matter of debate. MRI-Linac is a new frontier in radiation oncology as it allows a superior visualization of the real-time anatomy of the patient and the current highest level of adaptive radiotherapy.

Methods: We present data regarding a total of 100 fractions in 20 patients who underwent MRI-guided prostate SBRT for low-to-intermediate risk prostate cancer with or without spacer. Translational and rotational shifts were computed on the pre- and post-treatment MRI acquisitions referring to the delivery position for antero-posterior, latero-lateral and cranio-caudal direction, and assessed using the Mann-Whitney U-Test.

Results: All patients were treated with a five sessions schedule (35 Gy/5fx) using MRI-Linac for a median fraction treatment time of $50 \mathrm{~min}$ (range, 46-65). In the entire study sample, median rotational displacement was $0.1^{\circ}$ in cranio-caudal, $-0.002^{\circ}$ in latero-lateral and $0.01^{\circ}$ in antero-posterior direction; median translational shift was 0.11 $\mathrm{mm}$ in cranio-caudal, $-0.24 \mathrm{~mm}$ in latero-lateral and $-0.22 \mathrm{~mm}$ in antero-posterior. A significant difference between spacer and no-spacer patients in terms of rotational shifts in the antero-posterior direction $(p=0.033)$ was observed; also for translational shifts a positive trend was detected in antero-posterior direction $(p=0.07)$, although with no statistical significance. We observed statistically significant differences in the pre-treatment planning phase in favor of the spacer cohort for several rectum dose constraints: rectum V32Gy $<5 \%(p=0.001)$, V28 Gy $<10 \%(p=0.001)$ and V18Gy $<35 \%(p=0.039)$. Also for bladder V35 Gy $<1 c c$, the use of spacer provided a dosimetric advantage compared to the no-spacer subpopulation $(p=0.04)$. Furthermore, PTV V33.2Gy $>95 \%$ was higher in the spacer cohort compared to the no-spacer one $(p=0.036)$.

(Continued on next page)
\end{abstract}

\footnotetext{
* Correspondence: f.cuccia1@virgilio.it

${ }^{1}$ Advanced Radiation Oncology Deparment, Sacro Cuore Don Calabria Hospital, Negrar, Verona, Italy

Full list of author information is available at the end of the article
}

(c) The Author(s). 2020 Open Access This article is licensed under a Creative Commons Attribution 4.0 International License, which permits use, sharing, adaptation, distribution and reproduction in any medium or format, as long as you give appropriate credit to the original author(s) and the source, provide a link to the Creative Commons licence, and indicate if changes were made. The images or other third party material in this article are included in the article's Creative Commons licence, unless indicated otherwise in a credit line to the material. If material is not included in the article's Creative Commons licence and your intended use is not permitted by statutory regulation or exceeds the permitted use, you will need to obtain permission directly from the copyright holder. To view a copy of this licence, visit http://creativecommons.org/licenses/by/4.0/. The Creative Commons Public Domain Dedication waiver (http://creativecommons.org/publicdomain/zero/1.0/) applies to the data made available in this article, unless otherwise stated in a credit line to the data. 
(Continued from previous page)

Conclusion: In our experience, the application of rectal hydrogel spacer for prostate SBRT resulted in a significant impact on rotational antero-posterior shifts contributing to limit prostate intra-fraction motion. Further studies with larger sample size and longer follow-up are required to confirm this ideally favorable effect and to assess any potential impact on clinical outcomes.

Keywords: Prostate cancer, Stereotactic body radiotherapy, Organ motion, Mri-linac

\section{Introduction}

The use of hypofractionated radiotherapy for prostate cancer has globally widespread, being endorsed by international guidelines $[1,2]$. The radiobiological rationale for using higher doses per fraction in prostate cancer lies on the known low alpha-beta ratio of the tumor, estimated in $1.5 \mathrm{~Gy}$, which reflects a superior sensitivity to $>2 \mathrm{~Gy} /$ fractions in terms of biological effect [3].

In recent years, technological progress has allowed clinicians to deliver very high doses per fraction with higher levels of accuracy and confidence [4].

The adoption of extremely hypofractionated schedules is gaining attention in the scientific community, as a fast and effective treatment option for PC patients [5-8]. In fact, stereotactic body radiotherapy (SBRT) enables high dose irradiation of small volumes with a rapid dose falloff and a limited exposure of nearby healthy structures. The use of SBRT in prostate cancer has been reported by several experiences in the literature, with long-term data that report this technique as a safe and effective treatment for localized prostate cancer [9].

When extreme hypofractionation is adopted, a crucial aspect is represented by target motion management, in order to ensure not only the optimal target coverage, but also the improved sparing of surrounding organs at risk (OARs) [10].

To date, a large amount of data is available about the management of interfraction motion, consisting of the daily assessment of patient preparation in terms of bladder and rectum anatomy, which are the main factors influencing prostate shifts $[11,12]$.

On the contrary, the investigation of intrafraction motion is less reported in the literature and mainly based on the use of implanted fiducial markers, reporting that major drifts and transitory motions occur mainly in the antero-posterior and cranio-caudal directions [13-15].

Furthermore, although the available imaging consisting of in-room computed tomography proved to be a reliable tool for image guided-radiotherapy (IGRT), in some districts kilovoltage and/or megavoltage CT may offer a limited anatomy visualization due to a sub-optimal soft-tissues contrast [16-18].

Another tool potentially helpful in mitigating prostate motion is represented by rectal hydrogel spacer (SpaceOAR, Boston Scientific,Marlborough, MA, USA), a polyethylene- glycol gel device that is injected in the Denonvilliers fascia pushing forward the prostate in order to enlarge the distance with the anterior rectal wall, by creating an interspace estimated between 7 and $15 \mathrm{~mm}$ of extent [19].

In this scenario, the recent introduction of MR-guided linacs may represent a potential game-changer; first, by providing a superior anatomy imaging that allows a better identification of target volumes and OARs structures, also refining contouring accuracy [17]. Secondly, MRI-guidance may improve the quality of inter- and intra-fraction motion assessment. Especially for intrafraction motion, MR-linacs enable a refined evaluation of prostate shifts during treatment by means of pre- and post-treatment MRI acquisitions.

In October 2019 we have started at our department the clinical activity with Unity Elekta MR-linac (Elekta Unity, Stockholm, Sweden). Unity is a new platform for radiotherapy that conjugates a $7 \mathrm{MV}$ flattening filter free (FFF) linear accelerator with a 1.5 Tesla MR system. In accordance with an ongoing prospective observational study approved by the internal Ethical Committee, we have started MRI-Linac-based treatments also including SBRT for localized prostate cancer [20, 21].

Herein we present our preliminary report on intrafraction prostate motion data registered for the first 20 patients treated with MR-linac, with the aim to assess the potential impact of the use of hydrogel rectal spacer.

\section{Materials and methods}

The following results are derived from the prospective observational study ongoing at our institution, which was approved on April 2019 by the Local Ethical Committee. (MRI/LINAC n 23,748 ).

Herein we report the data concerning 20 patients who underwent MRI-guided stereotactic body radiotherapy for low-intermediate risk prostate cancer with or without rectal hydrogel spacer placement from October 2019 to January 2020.

Inclusion criteria for prostate SBRT were as follows: age $>18$ years, Karnofsky Performance Status $>70$, histologically proven low or intermediate risk prostate cancer according to National Comprehensive Cancer Network v.1.2020 classification, no radiological evidence of pathological lymph-nodes or distant metastases, no other malignant tumors in the last 5 years, International 
Prostate Symptoms Score (IPSS) < 15. Androgen deprivation therapy was allowed according to risk group.

Exclusion criteria were: prostate volume $>80 \mathrm{cc}$, previous prostate surgery or previous transurethral resection of prostate performed within 6 months from RT, any MRI contraindication (electronic devices, claustrophobia etc.), the inability to gain informed consent.

Hydrogel spacer placement was proposed as optional to all patients, and consisted of a minimally invasive procedure performed under local anesthesia. The Urology Surgeon implanted the spacer in the Denonvilliers fascia, and radiotherapy treatment was scheduled to start within 3 weeks from the surgical procedure.

\section{Radiotherapy protocol}

After consultation, all patients were educated for the imaging protocol which consisted at first of the acquisition of a $3 \mathrm{~mm}$ slice thickness pelvis-CT scan in supine position with knee-ankle immobilization devices, for dose calculation purposes. Afterwards, a T2- weighted pelvis MRI was acquired at the Unity MRI-Linac. For both imaging procedures, all patients were required to have a comfortably full bladder (by drinking $500 \mathrm{ml}$ of water before the exam) and an empty rectum (by self-administering a fleet enema).

As far as target volume delineation, in the case of lowrisk disease, the clinical target volume (CTV) was the prostate gland alone, while for intermediate risk disease, the entirety of the seminal vesicles was contoured as well. The planning target volume (PTV) was generated by adding a $5 \mathrm{~mm}$ margin in all directions, except for the posterior direction where a $3 \mathrm{~mm}$ expansion was applied, according to published studies [20, 21].

The rectum, bladder, penile bulb, urethra, and femoral heads were manually contoured as OARs.

The SBRT schedule consisted of five daily fractions of 7 Gy (total prescription dose, $D_{p}=35 \mathrm{~Gy}$ ) for all patients, equal to a normalized total dose of $2 \mathrm{~Gy}$ per fraction (NTD2) ranging between 70 and 85 Gy, assuming an $\alpha / \beta$ ratio between 3 and 1.5 Gy for PC.

The dose distribution normalization was calculated to ensure a minimum 95\% of the PTV to receive at least the $95 \%$ of the prescribed dose, and less than $2 \%$ of the PTV to receive $107 \%$ of Dp.

For OARs, the following constraints were applied: for the rectum: V18 Gy $\leq 35 \%$, V28 Gy $\leq 10 \%$, V32 Gy $\leq 5 \%$, Dmax $\leq 35$ Gy; for the bladder: Dmax $\leq 35$ Gy; no hotspots for the urethral PRV; for the intestinal loops Dmax $<32$ Gy. Dmax was always referred to the hottest $1 \mathrm{~cm}^{3}$ of the anatomical structure.

Static field intensity-modulated radiotherapy (IMRT) delivered with 16 beams were applied for generating baseline treatment plans.

Daily adapted radiotherapy is delivered with Unity using two alternative protocols: 'adapt-to-position' (ATP) and 'adapt-to-shape' (ATS). ATP is based on the daily isocenter position change in reference to the pre-treatment $\mathrm{CT}$. In the case of the ATS workflow, a re-contouring of the daily MRI is performed to adapt the treatment plan to the real-time anatomy of the patient. Thus, ATS allows clinicians to refine the dose delivery based on daily changes in the size, shape, and position of PTV and OARs.

More specifically, before each fraction, a new T2weighted MRI sequence (preMRI) is acquired and rigidly registered to the simulation MR. The original set of contours is transferred to the daily preMRI using deformable registration, and then edited, where needed, at physician's discretion. A full re-optimization is performed by the physicist and, during the second optimization phase (i.e. the segmentation phase), a second verification MRI scan is acquired to assess if bladder and rectum deformations can be considered negligible. In the case of unacceptable deformations, the patient was required to repeat the preparation protocol before being repositioned for treatment. Otherwise, the treatment is delivered using a cine-MRI, typically acquired on two coronal and sagittal planes to monitor patient motion. At the end of the session, a further post-MRi scan was performed, to estimate the intrafraction organ motion.

\section{Data collection and statistical analysis}

A manual re-contouring of the prostate CTV was performed by one physician in both pre- and post-MRI scans. Afterwards, a soft tissue-rigid registration with the daily planning MRI was generated based on the volume of interest (i.e. the prostate CTV). Then, translational and rotational shifts were registered in all directions (cranio-caudal, antero-posterior and latero-lateral) to assess intrafraction motion of the prostate referring to the delivery position.

Descriptive statistics were collected for continuous variables (median, maximum and minimum values and standard deviation). The Mann-Whitney U-test was used to compare the entity of translational and rotational shifts between the spacer and no-spacer cohorts, and the potential impact of prostate CTV volume on organ motion for the entire population. A $p$-value $<0.05$ was assumed to be statistically significant. All statistical analyses were performed with Graphpad Prism software v.8.4.2 (Graphpad Software, San Diego, CA, USA).

\section{Results}

The pre- and post-treatment MRI data regarding a total of 100 fractions in 20 consecutive patients who underwent MRI-guided prostate SBRT are herein presented. Ten patients were treated without hydrogel rectal spacer, and ten after the insertion of hydrogel spacer. This procedure was well tolerated in all patients, except in one 
case who developed rectal tenesmus fully resolved after local steroids. The median time for fraction was $50 \mathrm{~min}$ (range, 46-65 $\mathrm{min}$ ) for the entire cohort and the median CTV volume was $57.3 \mathrm{cc}$ (range, 25.3-74.3 cc). Median spacer interface was $0.99 \mathrm{~cm}$ (range, $0.44-1.49 \mathrm{~cm}$ ). Patients' characteristics and toxicity patterns are summarized, respectively in Table 1 and Table 2.

In the entire sample of the study, median rotational displacement was $0.1^{\circ}$ in cranio-caudal (X-axis), $-0.002^{\circ}$ (Y-axis) in latero-lateral and $0.01^{\circ}$ (Z-axis) in anteroposterior direction; median translational shift was 0.11 $\mathrm{mm}$ in cranio-caudal, $-0.24 \mathrm{~mm}$ in latero-lateral and $0.22 \mathrm{~mm}$ in antero-posterior.

For rotational shifts, the median displacement in craniocaudal direction (X-axis) was $0.18^{\circ}$ (range: $-0.37^{\circ} / 0.16^{\circ}$; $\mathrm{SD}=0.15^{\circ}$ ) for the no-spacer subgroup vs $0.11^{\circ}$ (range: $\left.0.70^{\circ} / 0.31 ; \mathrm{SD}=0.25^{\circ}\right)$ for the spacer subgroup $(p=0.108)$, in latero-lateral direction (Y-axis) we recorded a median displacement of $-0.04^{\circ}$ degrees (range: $-0.36^{\circ} / 0.82$; $\mathrm{SD}=$ $0.33^{\circ}$ ) in the no-spacer subgroup vs $-0.03^{\circ}$ degrees (range: $\left.-0.16^{\circ} / 0.13^{\circ} ; \mathrm{SD}=0.08^{\circ}\right)$ in the spacer subgroup $(p=0.78)$. A statistically significant difference was observed in the antero-posterior direction (Z-axis) between the spacer and the no-spacer cohorts $(p=0.033)$, with respective values of $-0.0005^{\circ}$ (range: $-0.30^{\circ} / 0.12$; $\mathrm{SD}=0.11^{\circ}$ ) and $0.09^{\circ}$ (range: $\left.-0.08^{\circ} / 0.26^{\circ} ; \mathrm{SD}=0.10^{\circ}\right)$.

Table 1 Baseline patients' characteristics

\begin{tabular}{|c|c|c|}
\hline \multicolumn{3}{|c|}{ Age, years (median, range): } \\
\hline SpaceOAR & $70(54-78)$ & \multirow[t]{2}{*}{$p=0.44$} \\
\hline No-SpaceOAR & $66(56-75)$ & \\
\hline \multicolumn{3}{|c|}{ PSA, ng/ml (median, range): } \\
\hline SpaceOAR & $9.3(6.6-19)$ & \multirow[t]{2}{*}{$p=0.08$} \\
\hline No-SpaceOAR & $6.8(4.2-12.7)$ & \\
\hline \multicolumn{3}{|l|}{ Risk Group (n / \%): } \\
\hline \multicolumn{3}{|c|}{ (Low/ Favorable Intermediate/Unfavorable Intermediate) } \\
\hline SpaceOAR & $3(30 \%) / 4(40 \%) / 3(30 \%)$ & \\
\hline No-SpaceOAR & $2(20 \%) / 6(60 \%) / 2(20 \%)$ & \\
\hline \multicolumn{3}{|c|}{ Androgen deprivation therapy $(\mathrm{n} / \%)$ : } \\
\hline SpaceOAR & $3(30 \%)$ & \\
\hline No-SpaceOAR & $2(20 \%)$ & \\
\hline \multicolumn{3}{|c|}{ Prostate Volume, cc (median, range): } \\
\hline SpaceOAR & $62.5(49.8-79)$ & \multirow[t]{2}{*}{$p=0.23$} \\
\hline No-SpaceOAR & $55.5(29.7-79)$ & \\
\hline \multicolumn{3}{|c|}{ Planning Target Volume, cc (median, range): } \\
\hline SpaceOAR & $118.8(85.7-150.1) p=0.17$ & \\
\hline No-SpaceOAR & $110.3(70.9-145.3)$ & \\
\hline \multicolumn{3}{|c|}{ IPSS score (median, range): } \\
\hline SpaceOAR & $7(0-15) \mathrm{s}$ & $p=0.14$ \\
\hline No-SpaceOAR & $5(0-10)$ & \\
\hline
\end{tabular}

Table 2 Acute Toxicity Rates (CTCAE v.5)

\begin{tabular}{ll}
\hline Genitourinary & G2 / G1 (n / \%) \\
\hline -SpaceOAR & $2(20 \%) / 2(20 \%)$ \\
-No-SpaceOAR & $1(10 \%) / 4(40 \%)$ \\
Gastrointestinal & G2 / G1 (n / \%) \\
-SpaceOAR & $0(0 \%) / 1(10 \%)$ \\
-No-SpaceOAR & $0(0 \%) / 1(10 \%)$ \\
\hline
\end{tabular}

As far as translational shifts, in cranio-caudal direction we recorded a median displacement of respectively 0.06 $\mathrm{mm}$ (range: $-1.07 \mathrm{~mm} / 0.89 \mathrm{~mm} ; \mathrm{SD}=0.46 \mathrm{~mm}$ ) and $0.42 \mathrm{~mm}$ (range: $-0.16 \mathrm{~mm} / 3.1 \mathrm{~mm}$; $\mathrm{SD}=1.25 \mathrm{~mm}$ ) in the no-spacer and spacer patients $(p=0.75)$, while for laterolateral direction median displacement values were -0.15 $\mathrm{mm}$ (range: $-2.5 \mathrm{~mm} / 4.1 \mathrm{~mm} ; \mathrm{SD}=2.5 \mathrm{~mm}$ ) and -0.24 $\mathrm{mm}$ (range: $-1.42 \mathrm{~mm} / 4.5 \mathrm{~mm}$; $\mathrm{SD}=2.5 \mathrm{~mm}$ ) for spacer and no-spacer cohorts $(p=0.77)$. In the Z-axis, the spacer subgroup reported a translational shift of $-0.42 \mathrm{~mm}$ (range: $-1.34 \mathrm{~mm} / 0.89 \mathrm{~mm} ; \mathrm{SD}=0.67 \mathrm{~mm}$ ) vs $-0.17 \mathrm{~mm}$ (range: $-2.65 \mathrm{~mm} / 0.60 \mathrm{~mm}$; $\mathrm{SD}=0.91 \mathrm{~mm}$ ) in the no-spacer subgroup $(p=0.07)$. (Table 3 and Fig. 1 ).

We have also investigated the potential impact of prostate CTV volume on organ motion, reporting no statistically significant correlation when comparing rotational and translational shifts of patients with a prostate CTV $>57.3 \mathrm{cc}$ or $<57.3 \mathrm{cc}$. (Table 4 and Fig. 2).

As far as the dosimetric analysis, we observed statistically significant differences in the pre-treatment planning phase in favor of the spacer cohort for several rectum dose constraints: rectum V32Gy $<5 \%(p=0.001), \mathrm{V} 28 \mathrm{~Gy}<10 \%$ $(p=0.001)$ and $\mathrm{V} 18 \mathrm{~Gy}<35 \%(p=0.039)$. Also for bladder V35 Gy $<1 \mathrm{cc}$, the use of spacer provided a dosimetric advantage compared to the no-spacer subpopulation $(p=$ 0.04). Furthermore, PTV V 33.2 Gy $>95 \%$ was higher in the spacer cohort compared to the no-spacer one $(p=0.036)$.

As far as the daily adapted planning, the use of rectal spacer was found to produce a dosimetric advantage in terms of rectum sparing and PTV coverage. More specifically, all the dose constraints used for rectum were significantly reduced as follows: rectum $\mathrm{V} 35 \mathrm{~Gy}<1 \mathrm{cc} \quad(p=0.0001)$, V32Gy $<5 \% \quad(p=0.00001)$, V28 Gy $<10 \% \quad(p<0.00001)$, V18Gy $<35 \%(p<0.00001)$. Similarly to the baseline planning phase, bladder V35 Gy $<1 \mathrm{cc}$ was found to be reduced in the spacer cohort $(p=0.00004)$. Notably, also the planning goals for tumor coverage (i.e. PTV V33.2Gy $>95 \%$ and PTV $37.5 \mathrm{~Gy}<2 \%$ ) were improved in the spacer group vs the no-spacer one ( $p=0.002$ and $p=0.0001$, respectively). (Table 5).

\section{Discussion}

The present paper depicts preliminary data of a total of 100 fractions in a series of 20 patients treated with MRguided SBRT for prostate cancer, including in the 
Table 3 Rotational and translational shifts with and without spacer

\begin{tabular}{|c|c|c|c|c|c|c|c|}
\hline & \multicolumn{3}{|l|}{ Spacer } & \multicolumn{3}{|c|}{ No Spacer } & \multirow[t]{2}{*}{$p$-value } \\
\hline & Median & SD & Range & Median & SD & Range & \\
\hline \multicolumn{8}{|c|}{ Rotational shifts (degrees) } \\
\hline AP & $-0.0005^{\circ}$ & $0.11^{\circ}$ & $-0.30^{\circ} / 0.12^{\circ}$ & $0.09^{\circ}$ & $0.10^{\circ}$ & $-0.08^{\circ} / 0.26^{\circ}$ & 0.033 \\
\hline LL & $-0.03^{\circ}$ & $0.08^{\circ}$ & $-0.16^{\circ} / 0.13^{\circ}$ & $-0.04^{\circ}$ & $0.33^{\circ}$ & $-0.36^{\circ} / 0.82^{\circ}$ & 0.78 \\
\hline CC & $0.11^{\circ}$ & $0.25^{\circ}$ & $-0.70^{\circ} / 0.31$ & $0.18^{\circ}$ & $0.15^{\circ}$ & $-0.37^{\circ} / 0.168^{\circ}$ & 0.108 \\
\hline \multicolumn{8}{|c|}{ Translational shifts (mm) } \\
\hline AP & -0.42 & 0.67 & $-1.34 / 0.89$ & -0.17 & 0.91 & $-2.65 / 0.6$ & 0.07 \\
\hline LL & -0.15 & 2.5 & $-2.5 / 4.1$ & -0.24 & 2.5 & $-1.42 / 4.5$ & 0.77 \\
\hline CC & -0.42 & 1.25 & $-0.16 / 3.1$ & 0.06 & 0.46 & $-1.07 / 0.89$ & 0.75 \\
\hline
\end{tabular}

AP Antero-posterior, CC Cranio-caudal, LL Latero-lateral, SD Standard deviation

sample 10 patients who received radiotherapy after the insertion of a rectal hydrogel spacer and 10 patients who were treated without spacer.

The implementation of rectal spacer in prostate SBRT is known to have a favorable impact on minimizing the dose to the anterior rectal wall, despite few data are currently available in terms of estimates of prostate movements during radiotherapy $[22,23]$.

Peri-rectal hydrogel spacer consists of a layer of polyethylene-glycol gel (SpaceOAR, Boston Scientific, Marlborough, MA, USA) implanted in the Denonvilliers' fascia in order to outdistance the prostate from anterior rectal wall. At the same time, this device is presumed to limit organ motion in the Z-axis, providing a sort of stabilization effect of the gland. To date, there is no clear evidence supporting the use of rectal spacer as a means to fixate prostate gland for the intrafraction motion, as some Authors hypothesize a potentially detrimental rectal wall inflammation [24].

Hedrick et al. reported in a series of 41 patients treated with proton therapy for prostate cancer with hydrogel spacer a significant advantage in terms of rectal sparing when compared to the dosimetric advantages provided by the use of endorectal balloons [25].
In agreement, in a previous report of our institution with conventional Linacs, we have also observed a dosimetric advantage provided by rectal gel in terms of anterior rectal wall exposure to low doses. Notably, in the abovementioned previous experience, the gel also improved the PTV coverage in terms of V95\% [19].

These advantages have also been recorded in the present experience in further support of the use of the hydrogel spacer in terms of superior rectal sparing and increased PTV coverage. Nonetheless, these data are preliminary and a longer follow-up is required to assess any potential impact on clinical outcomes and toxicity reports.

The role of hydrogel spacer on prostate motion patterns has been investigated in few previous experiences in the literature. Juneja et al. [26] reported in their series of 26 patients (respectively 12 with and 14 without spacer) a mean motion $>3 \mathrm{~mm}$ only in the $5 \%$ of delivered fractions, including also hypofractionated courses, while in our series we observed $>3 \mathrm{~mm}$ shifts in 2 cases (one in the X-and one in the Z-axis) among the spacer subgroup versus 3 cases in the no-spacer subgroup, where $>3 \mathrm{~mm}$ shifts were detected entirely in the latero-lateral direction. In another study, Picardi et al. [27] recorded

\section{Rotational Shifts}

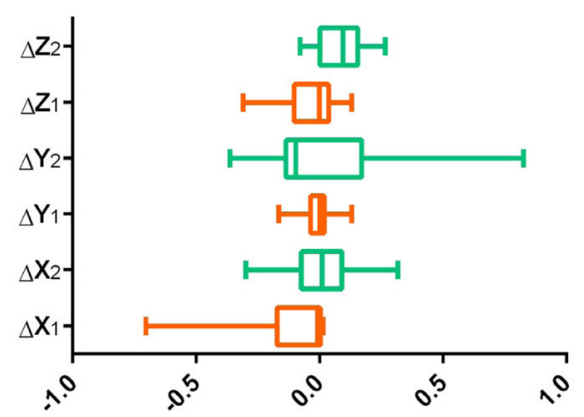

Translational Shifts

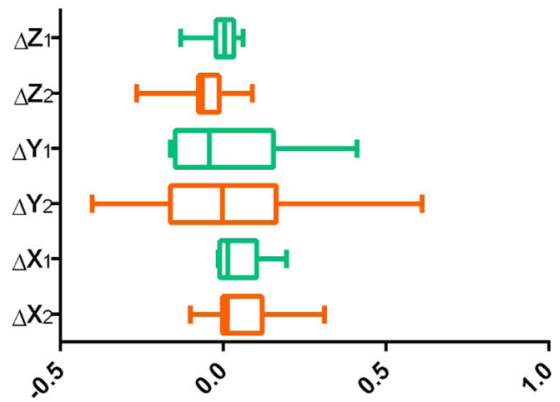

Fig. 1 Box plot graphics of rotational and translational shifts in all directions in patients with (1-orange) and without spacer (2-turquoise) 
Table 4 impact of prostate CTV volume on rotational and translational shifts

\begin{tabular}{ll}
\hline & $\begin{array}{l}C T V>57.3 c c \text { or } C T V<57.3 c c \\
p \text {-value }\end{array}$ \\
\hline Rotational shifts & \\
AP & 0.98 \\
LL & 0.69 \\
CC & 0.78 \\
Translational Shifts & \\
AP & 0.46 \\
LL & 0.61 \\
CC & 0.26 \\
\hline
\end{tabular}

in a cohort of 20 patients, treated with or without spacer, a displacement $>5 \mathrm{~mm}$ in latero-lateral, craniocaudal and antero-posterior direction respectively in the $0.8,6.5$ and $12.5 \%$ of cases, still not reaching a statistically significant difference between the two cohorts, unlike our series.

Similarly Pinkawa et al. [28] recorded a favorable effect provided by the use of rectal spacer not only in terms of outdistancing the prostate from the anterior rectal wall, but also in reducing larger posterior displacements when compared to patients treated without spacer insertion.

Finally, in a case report by Sumila et al. [29], a Cyberknife treatment for prostate SBRT recorded, over all the 5 treatment sessions, prostate shifts within $4 \mathrm{~mm}$ in all directions, regardless of the one hour-long treatment time. Despite referring to a single patient, this feature seems to support the stabilization effect on prostate motion, even in the case of longer treatment time techniques.

To the best of our knowledge, the present paper is the first analysis of prostate intra-fraction motion in MRguided SBRT comparing the outcomes of patients treated with or without rectal hydrogel spacer. Keeping in mind the small sample size of the present study, we hypothesize that the combination of MR-guided SBRT and the use of rectal hydrogel spacer may favorably impact on stereotactic radiotherapy for prostate cancer.
To date, we have no such longer follow-up to draw definitive conclusions on this matter, but it will surely be analyzed with larger data. Nonetheless, we believe that the combination of MRI-Linac and the use of SpaceOar hydrogel is feasible and well tolerated from patients, as reported in a previous report in which we have recorded no significant differences in terms of QoL between baseline and post-RT scores [21].

Despite being preliminary data, in our series we have observed a favorable impact of the use of hydrogel spacer in antero-posterior rotational and translational shifts, reporting a statistically significant impact on minimizing prostate displacement in the rotational antero-posterior direction $(p=0.033)$ and a positive trend for the anteroposterior translational shift $(p=0.07)$, although not reaching a statistically significant value.

The higher motion in the antero-posterior direction has also been reported in a fiducial markers-guided study by Kotte et al. [30] observing an intrafraction motion $>2 \mathrm{~mm}$ in radiotherapy sessions of $5-7 \mathrm{~min}$ in $66 \%$ of cases. Consequently, the Authors recommended a minimum $2 \mathrm{~mm}$ margin to keep into account the intrafraction motion, but larger margins are still required to cover any other potential uncertainty, as also described by other studies of prostate motion based on the use of fiducial markers [31].

Compared to other experiences investigating the role of spacer by means of Cone Beam CT imaging, MRILinacs provide a superior visualization of the patient's anatomy enabling clinicians to improve the accuracy in target volume delineation and to better distinguish the nearby healthy structures [32]. Furthermore, the adaptive workflow based on the daily re-planning based on the real-time anatomy refines the delivery process. Notably, these advantages may be theoretically counterbalanced by the longer treatment time for session, even if the potential impact of treatment time on organ motion still remains a matter of debate [33].

Nonetheless, the improved accuracy in real-time anatomy visualization along with a careful assessment of organ motion during the beam-on-time will be major
Rotational Shifts

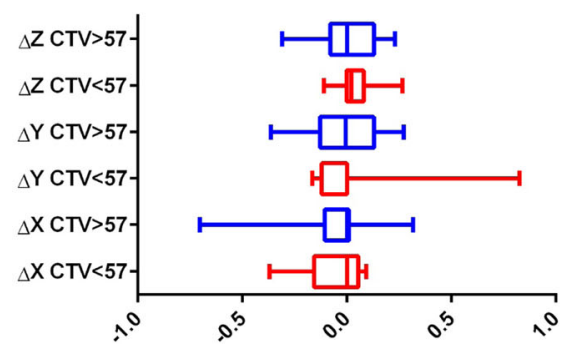

Translational Shifts

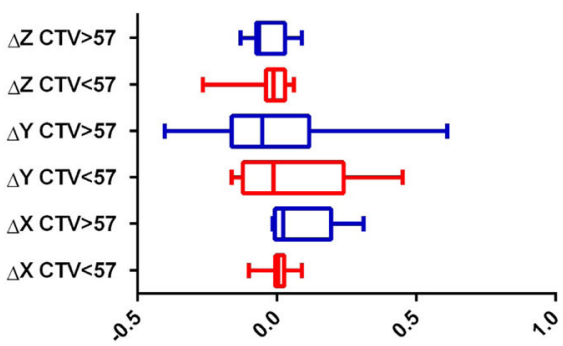

Fig. 2 Box plot graphics of rotational and translational shifts according to prostate CTV volume $>57$ cc (blue) or $<57$ cc (red) 
Table 5 Baseline planning and daily adaptive treatment sessions dosimetric data in patients with and without spacer

\begin{tabular}{|c|c|c|c|}
\hline Dosimetric Parameters & $\begin{array}{l}\text { SpaceOAR group } \\
\text { (mean } \pm \text { SD) }\end{array}$ & $\begin{array}{l}\text { No-SpaceOAR group } \\
\text { (mean } \pm \text { SD) }\end{array}$ & $p$ \\
\hline \multicolumn{4}{|l|}{ Baseline Planning: } \\
\hline \multicolumn{4}{|l|}{ Rectum } \\
\hline V35Gy < 1 cc & $0.01 \pm 0.02$ & $0.07 \pm 0.14$ & 0.21 \\
\hline V32Gy $<5 \%$ & $0.73 \pm 0.6$ & $3.28 \pm 0.9$ & 0.001 \\
\hline V28Gy < 10\% & $2.48 \pm 1.73$ & $7.88 \pm 0.98$ & 0.001 \\
\hline V18Gy < 35\% & $15.99 \pm 4.53$ & $19.98 \pm 2$ & 0.039 \\
\hline Rectal Volume (cc) & $72.6 \pm 39.8$ & $46 \pm 8$ & 0.06 \\
\hline \multicolumn{4}{|l|}{ Bladder } \\
\hline V35Gy $<1 \mathrm{cc}$ & $0.09 \pm 0.11$ & $0.24 \pm 0.21$ & 0.04 \\
\hline V30Gy (\%) & $7.09 \pm 4.90$ & $8.15 \pm 3.42$ & 0.24 \\
\hline V20Gy (\%) & $17.19 \pm 9.41$ & $19.7 \pm 6.74$ & 0.21 \\
\hline V10Gy (\%) & $36.25 \pm 12.89$ & $39.86 \pm 11.35$ & 0.43 \\
\hline V5Gy (\%) & $46.98 \pm 16.58$ & $54.85 \pm 16.22$ & 0.21 \\
\hline Bladder Volume (cc) & $390.9 \pm 182.8$ & $255.6 \pm 108.8$ & 0.06 \\
\hline \multicolumn{4}{|l|}{ Urethra } \\
\hline V35 Gy < 1 cc & $0.3 \pm 0.31$ & $0.33 \pm 0.25$ & 0.72 \\
\hline V33.2 Gy > 95\% & $100 \pm 0$ & $99.94 \% \pm 0.18 \%$ & 0.35 \\
\hline \multicolumn{4}{|l|}{ PTV } \\
\hline PTV37.5Gy < 2\% & $0.5 \pm 0.62$ & $0.74 \pm 0.67$ & 0.35 \\
\hline PTV33.2Gy > 95\% & $98.55 \pm 1.11$ & $96.8 \% \pm 1.25 \%$ & 0.036 \\
\hline PTV Volume (cc) & $121.8 \pm 20.9$ & $106.5 \pm 27$ & 0.17 \\
\hline \multicolumn{4}{|l|}{ Adaptive daily Planning: } \\
\hline \multicolumn{4}{|l|}{ Rectum } \\
\hline V35Gy < 1 cc & $0.01 \pm 0.03$ & $0.10 \pm 0.16$ & 0.0001 \\
\hline V32Gy < 5\% & $0.88 \pm 1$ & $3.43 \pm 1.33$ & 0.00001 \\
\hline V28Gy < 10\% & $2.95 \pm 2.46$ & $8.07 \pm 1.61$ & 0.00001 \\
\hline V18Gy < 35\% & $14.97 \pm 5.76$ & $20.81 \pm 2.59$ & 0.00001 \\
\hline Rectal Volume (cc) & $49.7 \pm 16.2$ & $47.7 \pm 10$ & 0.16 \\
\hline \multicolumn{4}{|l|}{ Bladder } \\
\hline V35Gy $<1$ cc & $0.04 \pm 0.09$ & $0.25 \pm 0.32$ & 0.00004 \\
\hline V30Gy (\%) & $6.98 \pm 2.79$ & $7.67 \pm 5.86$ & 0.43 \\
\hline V20Gy (\%) & $18.75 \pm 6.86$ & $19.33 \pm 10.41$ & 0.78 \\
\hline V10Gy (\%) & $40.06 \pm 13.17$ & $39.18 \pm 14.85$ & 0.70 \\
\hline V5Gy (\%) & $52.97 \pm 17.77$ & $53.37 \pm 19.97$ & 0.83 \\
\hline Bladder Volume (cc) & $215.4 \pm 166.9$ & $217.5 \pm 112.5$ & 0.17 \\
\hline \multicolumn{4}{|l|}{ Urethra } \\
\hline V35 Gy < 1 cc & $0.23 \pm 0.20$ & $0.22 \pm 0.17$ & 0.92 \\
\hline V33.2 Gy > 95\% & $99.98 \pm 0.13$ & $100 \% \pm 0 \%$ & 0.29 \\
\hline \multicolumn{4}{|l|}{ PTV } \\
\hline PTV37.5Gy < 2\% & $0.45 \pm 0.51$ & $1.39 \pm 1.05$ & 0.0001 \\
\hline PTV33.2Gy > 95\% & $97.91 \pm 1.35$ & $95.96 \% \pm 4.01 \%$ & 0.002 \\
\hline PTV Volume (cc) & $118.3 \pm 40.1$ & $111.4 \pm 35.5$ & 0.12 \\
\hline
\end{tabular}


assumptions to determine the proper margin expansion for radiotherapy treatment $[34,35]$, as in our series we recorded prostate displacement variation still within the expansion protocol of our Institution.

\section{Conclusion}

In the present study, the application of peri-rectal hydrogel spacer for prostate SBRT resulted in a statistically significant impact on rotational antero-posterior shifts compared to no-spacer cohort, contributing to limit prostate intra-fraction motion. Further studies with larger sample size and longer follow-up are required to confirm this ideally favorable effect and to assess any potential impact on clinical outcomes.

\section{Abbreviations}

ATP: Adapt-to-position; ATS: Adapt-to-shape; CT: Computed tomography; CTV: Clinical target volume; FFF: Flattening filter free; IGRT: Image guided radiotherapy; IMRT: Intensity modulated radiotherapy; IPSS: International prostate symptom score; MRI: Magnetic resonance imaging; NTD2: Normalized total dose at 2 Gy per fraction; OAR: Organ at risk; PC: Prostate cancer; PRV: Planning organ at risk volume; PTV: Planning target volume; SBRT: Stereotactic body radiotherapy; SD: Standard deviation

\section{Acknowledgments}

None.

\section{Authors' contributions}

Manuscript drafting: FC, RM, LN. Data collection and analysis: FC, MR, CV, BM, GS, ADS. Conception and study design: RM, FA, RR. Manuscript revision: FA, SC, RR, NGL, FR. Final approval: all authors.

\section{Funding}

No fundings.

\section{Availability of data and materials}

Not applicable.

\section{Ethics approval and consent to participate}

All patients signed an informed consent for the treatment.

\section{Consent for publication}

Not applicable.

\section{Competing interests}

Filippo Alongi is a consultant and speaker honoraria for Elekta and Boston Scientific; Ruggero Ruggieri is consultant for Elekta.

\section{Author details}

${ }^{1}$ Advanced Radiation Oncology Deparment, Sacro Cuore Don Calabria Hospital, Negrar, Verona, Italy. ${ }^{2}$ Urology Division, Sacro Cuore Don Calabria Hospital, Negrar, Verona, Italy. ${ }^{3}$ University of Brescia, Brescia, Italy.

Received: 27 May 2020 Accepted: 15 July 2020

Published online: 22 July 2020

\section{References}

1. Mottet N, Bellmunt J, Briers E, Bolla M, Bourke L, Cornford P, et al. EAU ESTRO - ESUR - SIOG guidelines on prostate cancer. Arnhem: Edn. presented at the EAU Annual Congress Amsterdam. 978-94-92671-07-3. Publisher: EAU guidelines officePlace published; 2020.

2. Morgan SC, Hoffman K, Loblaw DA, Buyyounouski MK, Patton C, Barocas D, et al. Hypofractionated radiation therapy for localized prostate cancer: executive summary of an ASTRO, ASCO and AUA evidence-based guideline. J Urol. 2019;201(3):528-34. https://doi.org/10.1097/JU.0000000000000071.

3. Brenner DJ, Martinez AA, Edmundson GK, Mitchell C, Thames HD, Armour EP. Direct evidence that prostate tumors show high sensitivity to fractionation (low alpha/beta ratio), similar to late-responding normal tissue. Int J Radiat Oncol Biol Phys. 2002;52(1):6-13. https://doi.org/10.1016/s03603016(01)02664-5.

4. De Bari B, Arcangeli S, Ciardo D, Mazzola R, Alongi F, Russi EG, et al. Extreme hypofractionation for early prostate cancer: biology meets technology. Cancer Treat Rev. 2016;50:48-60. https://doi.org/10.1016/j.ctrv.2016.08.005.

5. Brand DH, Tree AC, Ostler P, van der Voet H, Loblaw A, Chu W, et al. Intensity-modulated fractionated radiotherapy versus stereotactic body radiotherapy for prostate cancer (PACE-B): acute toxicity findings from an international, randomised, open-label, phase 3, non-inferiority trial. Lancet Oncol. 2019;20(11):1531-43. https://doi.org/10.1016/S1470-2045(19)30569-8.

6. Alongi F, Mazzola R, Fiorentino A, Corradini S, Aiello D, Figlia V, et al. Phase II study of accelerated Linac-based SBRT in five consecutive fractions for localized prostate cancer. Strahlenther Onkol. 2019;195(2):113-20. https:// doi.org/10.1007/s00066-018-1338-7.

7. Widmark A, Gunnlaugsson A, Beckman L, Thellenberg-Karlsson C, Hoyer M, Lagerlund $\mathrm{M}$, et al. Ultra-hypofractionated versus conventionally fractionated radiotherapy for prostate cancer: 5-year outcomes of the HYPO-RT-PC randomised, non-inferiority, phase 3 trial. Lancet. 2019;394(10196):385-95. https://doi.org/10.1016/S0140-6736(19)31131-6.

8. Nicosia L, Mazzola R, Rigo M, Figlia V, Giaj-Levra N, Napoli G, et al. Moderate versus extreme hypofractionated radiotherapy: a toxicity comparative analysis in low- and favorable intermediate-risk prostate cancer patients. J Cancer Res Clin Oncol. 2019;145(10):2547-54. https://doi.org/10.1007/ s00432-019-02983-3.

9. Jackson WC, Silva J, Hartman HE, Dess RT, Kishan AU, Beeler WH, et al. Stereotactic body radiation therapy for localized prostate cancer: a systematic review and meta-analysis of over 6,000 patients treated on prospective studies. Int J Radiat Oncol Biol Phys. 2019;104(4):778-89. https:// doi.org/10.1016/j.jirobp.2019.03.051.

10. De Bari B, Fiorentino A, Arcangeli S, Franco P, D'Angelillo RM, Alongi F. From radiobiology to technology: what is changing in radiotherapy for prostate cancer. Expert Rev Anticancer Ther. 2014;14(5):553-64. https://doi. org/10.1586/14737140.2014.883282.

11. Cubillos Mesías M, Boda-Heggemann J, Thoelking J, Lohr F, Wenz F, Wertz $\mathrm{H}$. Quantification and assessment of Interfraction setup errors based on cone beam CT and determination of safety margins for radiotherapy. PLoS One. 2016;11(3):e0150326. https://doi.org/10.1371/journal.pone.0150326.

12. Boda-Heggemann J, Lohr F, Wenz F, Flentje M, Guckenberger M. KV conebeam CT-based IGRT: a clinical review. Strahlenther Onkol. 2011;187(5):28491. https://doi.org/10.1007/s00066-011-2236-4.

13. Li HS, Chetty IJ, Enke CA, Foster RD, Willoughby TR, Kupellian PA, et al. Dosimetric consequences of intrafraction prostate motion. Int J Radiat Oncol Biol Phys. 2008;71(3):801-12. https://doi.org/10.1016/j. ijrobp.2007.10.049.

14. Kupelian P, Willoughby T, Mahadevan A, Djemil T, Weinstein G, Jani S, et al. Multi-institutional clinical experience with the calypso system in localization and continuous, real-time monitoring of the prostate gland during external radiotherapy. Int J Radiat Oncol Biol Phys. 2007;67(4):1088-98. https://doi. org/10.1016/j.jijobp.2006.10.026.

15. Langen KM, Willoughby TR, Meeks SL, Santhanam A, Cunningham A, Levine $L$, et al. Observations on real-time prostate gland motion using electromagnetic tracking. Int J Radiat Oncol Biol Phys. 2008;71(4):1084-90. https://doi.org/10.1016/j.jirobp.2007.11.054.

16. Lagendijk JJ, Raaymakers BW, Van den Berg CA, Moerland MA, Philippens ME, van Vulpen M. MR guidance in radiotherapy. Phys Med Biol. 2014;59(21): R349-69. https://doi.org/10.1088/0031-9155/59/21/R349.

17. Corradini S, Alongi F, Andratschke N, Belka C, Boldrini L, Cellini F, et al. MRguidance in clinical reality: current treatment challenges and future perspectives. Radiat Oncol. 2019;14(1):92. https://doi.org/10.1186/s13014019-1308-y.

18. Alongi F, de Crevoisier R, Corradini S, Créhange G, De Bari B. Daily IGRT for prostate cancer: can we stop the train? Radiother Oncol. 2018;128(2):389-90. https://doi.org/10.1016/j.radonc.2018.05.009.

19. Ruggieri R, Naccarato S, Stavrev P, Stavreva N, Fersino S, Giaj Levra N, et al. Volumetric-modulated arc stereotactic body radiotherapy for prostate cancer: dosimetric impact of an increased near-maximum target dose and of a rectal spacer. Br J Radiol. 2015;88(1054):20140736. https://doi.org/10. 1259/bjr.20140736

20. Mazzola R, Figlia V, Rigo M, et al. Feasibility and safety of 1.5 T MR-guided and daily adapted abdominal-pelvic SBRT for elderly cancer patients: 
geriatric assessment tools and preliminary patient-reported outcomes. J Cancer Res Clin Oncol. 2020. https://doi.org/10.1007/s00432-020-03230-w.

21. Alongi F, Rigo M, Figlia V, et al. 1.5 T MR-guided and daily adapted SBRT for prostate cancer: feasibility, preliminary clinical tolerability, quality of life and patient-reported outcomes during treatment. Radiat Oncol. 2020;15:69. https://doi.org/10.1186/s13014-020-01510-w.

22. Mok G, Benz E, Vallee JP, Miralbell R, Zilli T. Optimization of radiation therapy techniques for prostate cancer with prostate-rectum spacers: a systematic review. Int I Radiat Oncol Biol Phys. 2014;90:278-88.

23. Mariados N, Sylvester J, Shah D, Karsh L, Hudes R, Beyer D, et al. Hydrogel spacer prospective multicenter randomized controlled pivotal trial: dosimetric and clinical effects of perirectal spacer application in men undergoing prostate image guided intensity modulated radiation therapy. Int J Radiat Oncol Biol Phys. 2015;92:971-7.

24. Pinkawa M. Current role of spacers for prostate cancer radiotherapy. World J Clin Oncol. 2015;6(6):189-93. https://doi.org/10.5306/wjco.v6.16.189.

25. Hedrick SG, Fagundes M, Robison B, Blakey M, Renegar J, Artz M, et al. A comparison between hydrogel spacer and endorectal balloon: an analysis of intrafraction prostate motion during proton therapy. J Appl Clin Med Phys. 2017;18(2):106-12. https://doi.org/10.1002/acm2.12051.

26. Juneja P, Kneebone A, Booth JT, Thwaites DI, Kaur R, Colvill E, et al. Prostate motion during radiotherapy of prostate cancer patients with and without application of a hydrogel spacer: a comparative study. Radiat Oncol. 2015; 10:215. https://doi.org/10.1186/s13014-015-0526-1.

27. Picardi C, Rouzaud M, Kountouri M, Lestrade L, Vallée JP, Caparrotti F, et al. Impact of hydrogel spacer injections on interfraction prostate motion during prostate cancer radiotherapy. Acta Oncol. 2016;55(7):834-8. https:// doi.org/10.3109/0284186X.2015.1128118.

28. Pinkawa M, Piroth MD, Holy R, Escobar-Corral N, Caffaro M, Djukic V, et al. Spacer stability and prostate position variability during radiotherapy for prostate cancer applying a hydrogel to protect the rectal wall. Radiother Oncol. 2013;106:220-4.

29. Sumila M, Mack A, Schneider U, Storelli F, Curschmann J, Gruber G. Longterm intra-fractional motion of the prostate using hydrogel spacer during Cyberknife(R) treatment for prostate cancer-a case report. Radiat Oncol. 2014;9:186.

30. Kotte AN, Hofman P, Lagendijk JJ, van Vulpen M, van der Heide UA. Intrafraction motion of the prostate during external-beam radiation therapy: analysis of 427 patients with implanted fiducial markers. Int J Radiat Oncol Biol Phys. 2007;69(2):419-25. https://doi.org/10.1016/j.jprobp.2007.03.029.

31. O'Neill AG, Jain S, Hounsell AR, O'Sullivan JM. Fiducial marker guided prostate radiotherapy: a review. Br J Radiol. 2016;89(1068):20160296. https:// doi.org/10.1259/bjr.20160296.

32. Kerkhof EM, van der Put RW, Raaymakers BW, van der Heide UA, Jürgenliemk-Schulz IM, Lagendijk JJ. Intrafraction motion in patients with cervical cancer: the benefit of soft tissue registration using MRI. Radiother Oncol. 2009;93(1):115-21. https://doi.org/10.1016/j.radonc.2009.07.010.

33. Both S, Wang KK, Plastaras JP, Deville C, Bar Ad V, Tochner Z, et al. Real-time study of prostate intrafraction motion during external beam radiotherapy with daily endorectal balloon. Int J Radiat Oncol Biol Phys. 2011;81(5):13029. https://doi.org/10.1016/j.jijrobp.2010.08.052.

34. Jeong S, Lee JH, Chung MJ, Lee SW, Lee JW, Kang DG, et al. Analysis of geometric shifts and proper setup-margin in prostate cancer patients treated with pelvic intensity-modulated radiotherapy using Endorectal ballooning and daily enema for prostate immobilization. Medicine (Baltimore). 2016;95(2):e2387. https://doi.org/10.1097/MD.0000000000002387.

35. de Muinck Keizer DM, Kontaxis C, Kerkmeijer LGW, van der Voort van Zyp JRN, van den Berg CAT, Raaymakers BW, et al. Dosimetric impact of softtissue based intrafraction motion from 3D cine-MR in prostate SBRT. Phys Med Biol. 2020;65(2):025012. https://doi.org/10.1088/1361-6560/ab6241.

\section{Publisher's Note}

Springer Nature remains neutral with regard to jurisdictional claims in published maps and institutional affiliations.

\section{Ready to submit your research? Choose BMC and benefit from}

- fast, convenient online submission

- thorough peer review by experienced researchers in your field

- rapid publication on acceptance

- support for research data, including large and complex data types

- gold Open Access which fosters wider collaboration and increased citations

- maximum visibility for your research: over $100 \mathrm{M}$ website views per year

At BMC, research is always in progress.

Learn more biomedcentral.com/submissions 